PROCEEDINGS OF THE

AMERICAN MATHEMATICAL SOCIETY

Volume 133, Number 3, Pages 687-692

S 0002-9939(04)07648-8

Article electronically published on September 20, 2004

\title{
ON TENSOR PRODUCTS OF $k$-VERY AMPLE LINE BUNDLES
}

\author{
YUKITOSHI HINOHARA, KAZUYOSHI TAKAHASHI, AND HIROYUKI TERAKAWA
}

(Communicated by Michael Stillman)

\begin{abstract}
In this paper, we show that the tensor product of $a$-very ample and $b$-very ample line bundles on a complete algebraic variety is $(a+b)$-very ample.
\end{abstract}

\section{INTRODUCTION}

Let $X$ be a complete algebraic variety over an algebraically closed field $\mathrm{k}$ and $\mathcal{L}$ a line bundle on $X$. For a nonnegative integer $k \geq 0, \mathcal{L}$ is said to be $k$-very ample if the restriction map $\Gamma(X, \mathcal{L}) \rightarrow \Gamma\left(X, \mathcal{L} \otimes \mathcal{O}_{Z}\right)$ is surjective for every 0-dimensional subscheme $Z$ of length $k+1$. Note that $\mathcal{L}$ is 0 -very ample if and only if it is generated by its global sections, and that $\mathcal{L}$ is 1 -very ample if and only if it is very ample.

Beltrametti, Sommese, and others have studied "higher order embeddings", e.g., $k$-spannedness, $k$-very ampleness and $k$-jet ampleness of polarized manifolds (see [2], 3], 4], [5], 6] and so on).

In this paper, we study the tensor products of $k$-very ample line bundles on $X$. As is well known, if $\mathcal{L}_{1}$ and $\mathcal{L}_{2}$ are $a$-spanned and $b$-spanned respectively, then $\mathcal{L}_{1} \otimes \mathcal{L}_{2}$ is $(a+b)$-spanned (see [3, Lemma 0.5.3] and 1, Lemma 1.1.3]). Also, if $\mathcal{L}_{1}$ and $\mathcal{L}_{2}$ are $a$-jet ample and $b$-jet ample respectively, then $\mathcal{L}_{1} \otimes \mathcal{L}_{2}$ is $(a+b)$-jet ample (see [5, Lemma 2.2]). However, it seems to be not clear whether this is the case for $k$-very ampleness or not (see [6, (0.3)]). The purpose of the paper is to give the affirmative answer for the question. Our main result is as follows.

Theorem 1.1. Let $X$ be a complete algebraic variety over an algebraically closed field $\mathrm{k}$ and $\mathcal{L}_{1}, \mathcal{L}_{2}$ line bundles on $X$. Let $a, b$ be nonnegative integers. If $\mathcal{L}_{1}$ is a-very ample and $\mathcal{L}_{2}$ is b-very ample, then $\mathcal{L}_{1} \otimes \mathcal{L}_{2}$ is $(a+b)$-very ample.

We prove the theorem by induction on the length of 0 -dimensional subschemes of $X$. The idea is to see the socle in an Artinian ring and to construct 0-dimensional subschemes of smaller length from the given 0-dimensional scheme.

Received by the editors December 21, 2000 and, in revised form, November 9, 2003.

2000 Mathematics Subject Classification. Primary 14E25, 14C20; Secondary 13E10.

Key words and phrases. $k$-very ampleness, tensor product, 0 -dimensional scheme, socle. 


\section{Preliminaries}

Let $Z$ be a 0 -dimensional subscheme of $X$. The support of $Z$, denoted by $|Z|$, is the underlying topological space of the reduced scheme $Z_{\text {red }}$. The length of $Z$ is defined to be the dimension of the vector space $\Gamma\left(X, \mathcal{O}_{Z}\right)$.

2.1. $k$-very ampleness and Artinian rings. Let $Z$ be a 0 -dimensional subscheme of $X$ of length $l$ and $|Z|=\left\{x_{1}, \ldots, x_{r}\right\}$. Assume that $Z$ is defined by the ideal sheaf $\mathcal{J} \subset \mathcal{O}_{X}$, and then $\mathcal{O}_{X} / \mathcal{J} \cong \prod_{i=1}^{r} \mathcal{O}_{X, x_{i}} / \mathcal{J}_{x_{i}}$ and $\sum_{i=1}^{r} \ell\left(\mathcal{O}_{X, x_{i}} / \mathcal{J}_{x_{i}}\right)=$ $l$. First note that the restriction map

$$
e_{\mathcal{L}, Z}: \Gamma(X, \mathcal{L}) \rightarrow \Gamma\left(X, \mathcal{L} \otimes \mathcal{O}_{Z}\right)
$$

factors through $\prod_{i=1}^{r} \mathcal{L}_{x_{i}}$, that is, we have the following commutative diagram:

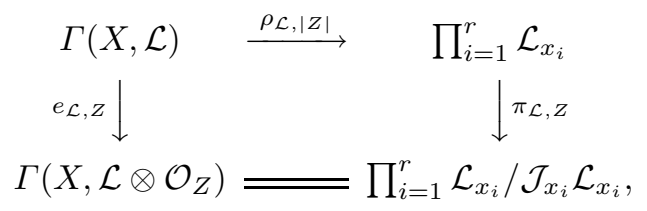

where $\rho_{\mathcal{L},|Z|}$ sends $s \in \Gamma(X, \mathcal{L})$ to $\left(s_{x_{1}}, \ldots, s_{x_{r}}\right) \in \prod_{i=1}^{r} \mathcal{L}_{x_{i}}$. Here note that $\pi_{\mathcal{L}, Z}$ is surjective.

Let $A_{Z}=\prod_{i=1}^{r} \mathcal{O}_{X, x_{i}}, V_{Z}:=\rho_{\mathcal{L},|Z|}(\Gamma(X, \mathcal{L}))$, and let $J_{Z}$ be the ideal of $A_{Z}$ such that $A_{Z} / J_{Z}=\prod_{i=1}^{r} \mathcal{O}_{X, x_{i}} / \mathcal{J}_{x_{i}}$. From now on, we will identify $\prod_{i=1}^{r} \mathcal{L}_{x_{i}}$ with $A_{Z}$ and $\prod_{i=1}^{r} \mathcal{L}_{x_{i}} / \mathcal{J}_{x_{i}} \mathcal{L}_{x_{i}}$ with $A_{Z} / J_{Z}$ respectively. Then we obtain the following.

Lemma 2.1. $e_{\mathcal{L}, Z}$ is surjective if and only if $V_{Z}+J_{Z}=A_{Z}$.

Proof. If $e_{\mathcal{L}, Z}$ is surjective, then for any element $a \in A_{Z}$ there exists a section $s \in \Gamma(X, \mathcal{L})$ such that $\pi_{\mathcal{L}, Z}(a)=e_{\mathcal{L}, Z}(s)$. We have $\pi_{\mathcal{L}, Z}\left(a-\rho_{\mathcal{L},|Z|}(s)\right)=0$, and $a-\rho_{\mathcal{L},|Z|}(s) \in J_{Z}$. Therefore $V_{Z}+J_{Z}=A_{Z}$.

Suppose that $V_{Z}+J_{Z}=A_{Z}$. It follows from the above diagram that, for any section $t \in \Gamma\left(X, \mathcal{L} \otimes \mathcal{O}_{Z}\right)$, there exists an element $b \in A_{Z}$ such that $\pi_{\mathcal{L}, Z}(b)=t$. Since $V_{Z}+J_{Z}=A_{Z}$, we can write $b=\rho_{\mathcal{L},|Z|}(s)+f$ for some $s \in \Gamma(X, \mathcal{L})$ and $f \in J_{Z}$. Then

$$
e_{\mathcal{L}, Z}(s)=\pi_{\mathcal{L}, Z}\left(\rho_{\mathcal{L},|Z|}(s)\right)=\pi_{\mathcal{L}, Z}(b-f)=\pi_{\mathcal{L}, Z}(b)=t .
$$

Therefore $e_{\mathcal{L}, Z}$ is surjective.

From the definition of $k$-very ampleness and the above lemma, we obtain

Proposition 2.2. With the above notation, $\mathcal{L}$ is $k$-very ample if and only if $V_{Z}+$ $J_{Z}=A_{Z}$ for any 0 -dimensional subscheme $Z \subset X$ of length $k+1$.

2.2. $k$-very ampleness and finite sets. Let $S=\left\{x_{1}, \ldots, x_{r}\right\}$ be a finite set of $r$ points of $X$, and let $Z$ be a 0 -dimensional subscheme of $X$ with the defining ideal sheaf $\mathcal{I} \subset \mathcal{O}_{X}$ such that $|Z| \subseteq S$. We may assume that $|Z|=\left\{x_{1}, \ldots, x_{t}\right\}$ for $t \leq r$. Consider the following commutative diagram (with the identification noted above):

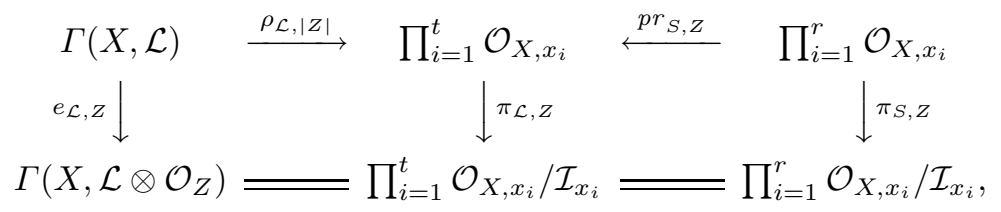


where $\mathcal{I}_{x_{i}}=\mathcal{O}_{X, x_{i}}$ for $i=t+1, \ldots, r$ and $p r_{S, Z}$ is the projection. We define

$$
\rho_{\mathcal{L}, S}: \Gamma(X, \mathcal{L}) \longrightarrow \prod_{i=1}^{r} \mathcal{O}_{X, x_{i}}
$$

by sending $s \in \Gamma(X, \mathcal{L})$ to $\left(s_{x_{1}}, \ldots, s_{x_{r}}\right) \in \prod_{i=1}^{r} \mathcal{O}_{X, x_{i}}$, and then $\operatorname{pr}_{S, Z} \circ \rho_{\mathcal{L}, S}=$ $\rho_{\mathcal{L},|Z| \cdot}$

Let $A_{S}=\prod_{i=1}^{r} \mathcal{O}_{X, x_{i}}, V_{S}=\rho_{\mathcal{L}, S}(\Gamma(X, \mathcal{L}))$, and let $I$ be the ideal of $A_{S}$ such that $A_{S} / I=\prod_{i=1}^{r} \mathcal{O}_{X, x_{i}} / \mathcal{I}_{x_{i}}$. Then we obtain the following lemma by the same argument as in the proof of Lemma 2.1

Lemma 2.3. With the above notation, $e_{\mathcal{L}, Z}$ is surjective if and only if $V_{S}+I=A_{S}$ in $A_{S}$.

Remark 2.4. In the above lemma, note that $A_{S}$ and $V_{S}$ do not depend on the 0-dimensional subscheme $Z$ but on the finite set $S$.

\section{Proof of Theorem 1.1}

Let $Z$ be a 0 -dimensional subscheme of $X$ of length $a+b+1,|Z|=\left\{x_{1}, \ldots, x_{r}\right\}$, and let $\mathcal{J} \subset \mathcal{O}_{X}$ be the defining ideal sheaf of $Z$. Put $A=\prod_{i=1}^{r} \mathcal{O}_{X, x_{i}}$ and let $J$ be the ideal of $A$ such that $A / J=\prod_{i=1}^{r} \mathcal{O}_{X, x_{i}} / \mathcal{J}_{x_{i}}$, and then

$$
\ell(A / J)=\sum_{i=1}^{r} \ell\left(\mathcal{O}_{X, x_{i}} / \mathcal{J}_{x_{i}}\right)=a+b+1 .
$$

Let $\rho_{\mathcal{L}_{i},|Z|}: \Gamma\left(X, \mathcal{L}_{i}\right) \rightarrow A$ for $i=1,2$ and $\rho_{\mathcal{L}_{1} \otimes \mathcal{L}_{2},|Z|}: \Gamma\left(X, \mathcal{L}_{1} \otimes \mathcal{L}_{2}\right) \rightarrow A$ be the maps defined above. Then we have the following commutative diagram:

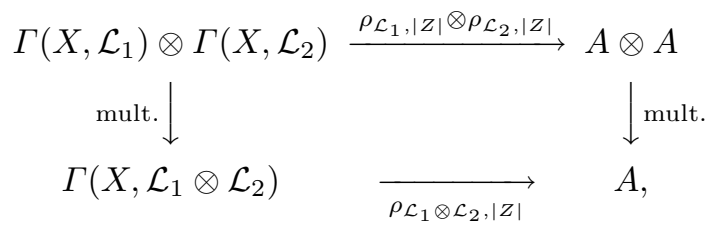

where each mult. is the multiplication map. Hence we obtain

$$
V_{1} V_{2} \subset V
$$

where $V_{i}:=\rho_{\mathcal{L}_{i},|Z|}\left(\Gamma\left(X, \mathcal{L}_{i}\right)\right)$ for $i=1,2, V:=\rho_{\mathcal{L}_{1} \otimes \mathcal{L}_{2},|Z|}\left(\Gamma\left(X, \mathcal{L}_{1} \otimes \mathcal{L}_{2}\right)\right)$, and $V_{1} V_{2}$ is the set of all elements of the form $\sum_{i} u_{i} v_{i}\left(u_{i} \in V_{1}, v_{i} \in V_{2}\right)$.

Since $\mathcal{L}_{1}$ is $a$-very ample, $e_{\mathcal{L}_{1}, Z^{\prime}}$ is surjective for any 0 -dimensional subscheme $Z^{\prime} \subseteq Z$ of length $a+1$, and from Lemma 2.3 we see that $V_{1}+I=A$ for any ideal $I$ of $A$ such that $\ell(A / I)=a+1$. Similarly, $V_{2}+I^{\prime}=A$ for any ideal $I^{\prime}$ of $A$ such that $\ell\left(A / I^{\prime}\right)=b+1$. In order to prove that $\mathcal{L}_{1} \otimes \mathcal{L}_{2}$ is $(a+b)$-very ample, by Proposition 2.2, we have to show that $V+J=A$, hence $V_{1} V_{2}+J=A$ from (3.1). Therefore it is sufficient to prove the following claim.

Claim 3.1. Let $\left(A_{i}, \mathfrak{m}_{i}, \mathrm{k}\right)$ be local rings, let $A=\prod_{i=1}^{r} A_{i}$ be the direct product, and let $V_{1}, V_{2}$ be $\mathrm{k}$-vector spaces contained in $A$. Assume that $A$ contains $\mathrm{k}$ and $V_{1}+I=A\left(\right.$ resp. $\left.V_{2}+I^{\prime}=A\right)$ for any ideal $I$ (resp. $\left.I^{\prime}\right)$ of A such that $\ell(A / I)=a+1$ (resp. $\left.\ell\left(A / I^{\prime}\right)=b+1\right)$. Then $V_{1} V_{2}+J=A$ for any ideal $J$ of $A$ such that $\ell(A / J)=a+b+1$, where $V_{1} V_{2}$ is the set of all elements of the form $\sum_{i} u_{i} v_{i}$ $\left(u_{i} \in V_{1}, v_{i} \in V_{2}\right)$. 
Proof. Let $1_{i}$ be the identity element of $A_{i}$ for $i=1, \ldots, r$. We identify $1_{i}$ with the element $\left(0, \ldots, 0,1_{i}, 0, \ldots, 0\right) \in A$. Then $1_{1}, \ldots, 1_{r}$ are orthogonal idempotents of $A$, that is, $1_{i}^{2}=1_{i}$ for each $i, 1_{i} 1_{j}=0$ if $i \neq j$, and $\sum_{i=1}^{r} 1_{i}=1_{A}$.

We prove the claim by induction on $a$ and $b$.

Assume that $a=0$. If $I$ is an ideal of $A$ such that $\ell(A / I)=1$, then $I$ is of the form

$$
I=A_{1} \times \cdots \times A_{i-1} \times \mathfrak{m}_{i} \times A_{i+1} \times \cdots \times A_{r}
$$

for some $i$. Since $V_{1}+I=A$ by the assumption, there exist $v_{1} \in V_{1}, m_{i} \in \mathfrak{m}_{i}$, and $c_{i} \in \prod_{j \neq i} A_{j}$ such that $v_{1}+m_{i}+c_{i}=1_{i}$. Hence we obtain $v_{1}=1_{i}-m_{i}-c_{i}$.

If $J$ is any ideal such that $\ell(A / J)=b+1$, then $V_{2}+J=A$ by the assumption, and in particular we may assume that $A_{i} \subset V_{2}+J$ for any $i$.

Now we show that $V_{1} V_{2}+J=A$. To do this, it is sufficient to prove $A_{i} \subset V_{1} V_{2}+J$ for all $i$. By the above assumption, we have

$$
v_{1} A_{i} \subset v_{1} V_{2}+v_{1} J, \quad \text { and } \quad v_{1} A_{i}=\left(1_{i}-m_{i}-c_{i}\right) A_{i}=A_{i}
$$

since $c_{i} A_{i}=0$ and $1_{i}-m_{i}$ is a unit in $A_{i}$. Therefore we obtain

$$
A_{i}=v_{1} A_{i} \subset v_{1} V_{2}+v_{1} J \subset V_{1} V_{2}+J .
$$

Similarly we can prove the case $b=0$.

Next assume that $a>0$ and $b>0$. Then the induction assumption is: (A1) if $a^{\prime} \leq a, b^{\prime} \leq b, a^{\prime}+b^{\prime}<a+b$, and $J^{\prime}$ is any ideal of $A$ such that $\ell\left(A / J^{\prime}\right)=a^{\prime}+b^{\prime}+1$, then $V_{1} V_{2}+J^{\prime}=A$.

This is equivalent to the assumption:

$(A 2)$ if $J^{\prime \prime}$ is any ideal of $A$ such that $\ell\left(A / J^{\prime \prime}\right)<a+b+1$, then $V_{1} V_{2}+J^{\prime \prime}=A$. Indeed, there are nonnegative integers $a^{\prime}, b^{\prime}$ such that $a^{\prime} \leq a, b^{\prime} \leq b, a^{\prime}+b^{\prime}<a+b$, and $\ell\left(A / J^{\prime \prime}\right)=a^{\prime}+b^{\prime}+1$. From the assumption $(A 1)$, we see that $V_{1} V_{2}+J^{\prime \prime}=A$. We will use this assumption repeatedly.

Let $J$ be an ideal of $A$ such that $\ell(A / J)=a+b+1$. Now we write $A$ (resp. $\mathfrak{m}$ ) instead of $A / J$ (resp. $\mathfrak{m} / J$ ) for simplicity, and we show that, if $\ell(A)=a+b+1$, then $V_{1} V_{2}=A$. To do this, we recall the definition of the socle of $A$. The socle of $A$ is defined to be the sum of all the ideals of length 1 of $A$ and is denoted by $\operatorname{Soc}(A)$.

Case 1. Assume that $\operatorname{dim}_{\mathrm{k}}(\operatorname{Soc}(A)) \geq 2$. Let $\mathfrak{n}_{1}, \mathfrak{n}_{2}$ be any two distinct minimal ideals in $\operatorname{Soc}(A)$. Since $\ell\left(A / \mathfrak{n}_{2}\right)<a+b+1$, it follows from assumption $(A 2)$ that $V_{1} V_{2}+\mathfrak{n}_{2}=A$. For any nonzero element $x$ of $\mathfrak{n}_{1}$, there exist elements $v \in V_{1} V_{2}$ and $y \in \mathfrak{n}_{2}$ such that $v+y=x$. Here note that $v \neq 0$ since $\mathfrak{n}_{1} \cap \mathfrak{n}_{2}=0$. Let $\mathfrak{n}^{\prime}$ be the ideal of $A$ generated by $v$. Then $\ell\left(A / \mathfrak{n}^{\prime}\right)<a+b+1$ and $\mathfrak{n}^{\prime}=A \cdot v=\mathrm{k} \cdot v \subset V_{1} V_{2}$ since $v=x-y \in \operatorname{Soc}(A)$. Therefore by assumption $(A 2)$, we obtain

$$
A=V_{1} V_{2}+\mathfrak{n}^{\prime}=V_{1} V_{2} \text {. }
$$

Case 2. Assume that $\operatorname{dim}_{\mathrm{k}}(\operatorname{Soc}(A))=1$. Then $A$ is a local ring and has the unique minimal ideal $\mathfrak{n}$. Since $\ell(A / \mathfrak{n})<a+b+1$, by assumption $(A 2)$, we obtain

$$
V_{1} V_{2}+\mathfrak{n}=A \text {. }
$$

It follows from [8. Theorem 221] that $A$ is a 0 -dimensional Gorenstein ring. For any ideal $\mathfrak{a}$ of the 0 -dimensional Gorenstein $\operatorname{ring} A$, we obtain $(0:(0: \mathfrak{a}))=\mathfrak{a}$ by 7, Exercise 3.2.15], and

$$
\ell((0: \mathfrak{a}))=\ell(A / \mathfrak{a}) \quad \text { and } \quad \ell(\mathfrak{a})=\ell(A /(0: \mathfrak{a}))
$$

by considering the length of the composition series. 
Let $\mathfrak{b}, \mathfrak{c}$ be any ideals of $A$ such that $\mathfrak{b} \supset \mathfrak{c}, \ell(\mathfrak{b})=b+1$, and $\ell(\mathfrak{c})=b$. From (3.2), we obtain $\ell(A /(0: \mathfrak{b}))=b+1$ and $\ell(A / \mathfrak{c})=a+1$. By the assumption of the claim, $V_{2}+(0: \mathfrak{b})=A$, and for any element $x \in(0: \mathfrak{c})$, there exist $v_{2} \in V_{2}$ and $z \in(0: \mathfrak{b})$ such that $v_{2}+z=x$. Similarly, $V_{1}+\mathfrak{c}=A$, and for any element $y \in \mathfrak{b}$, there exist $v_{1} \in V_{1}$ and $w \in \mathfrak{c}$ such that $v_{1}+w=y$. Then

$$
v_{1} v_{2}=(y-w)(x-z)=y x-y z-w x+w z=x y .
$$

Indeed, $y z \in \mathfrak{b} \cdot(0: \mathfrak{b})=0, w x \in \mathfrak{c} \cdot(0: \mathfrak{c})=0$, and $w z \in \mathfrak{c} \cdot(0: \mathfrak{b}) \subset \mathfrak{b} \cdot(0: \mathfrak{b})=0$. Hence we obtain

$$
(0: \mathfrak{c}) \cdot \mathfrak{b} \subset V_{1} V_{2} .
$$

Moreover $(0: \mathfrak{c}) \supsetneq(0: \mathfrak{b})$, and then $(0: \mathfrak{c}) \cdot \mathfrak{b} \neq 0$. We obtain

$$
\mathfrak{n} \subset(0: \mathfrak{c}) \cdot \mathfrak{b}
$$

because $\mathfrak{n}$ is the unique minimal ideal of $A$. Therefore by (3.3) and (3.4) we obtain $\mathfrak{n} \subset V_{1} V_{2}$, and then

$$
A=V_{1} V_{2}+\mathfrak{n}=V_{1} V_{2} .
$$

This completes the proof of the claim, hence Theorem 1.1.

We obtain the following corollary inductively.

Corollary 3.2. Let $X$ be a complete algebraic variety over an algebraically closed field $\mathrm{k}$ and $\mathcal{L}_{1}, \ldots, \mathcal{L}_{m}$ line bundles on $X$. Let $a_{1}, \ldots, a_{m}$ be nonnegative integers. If $\mathcal{L}_{i}$ is $a_{i}$-very ample for $i=1, \ldots, m$, then $\mathcal{L}_{1} \otimes \cdots \otimes \mathcal{L}_{m}$ is $\left(a_{1}+\cdots+a_{m}\right)$-very ample.

\section{ADDED IN PROOF}

After the paper was accepted for publication, we found that there is an error in the proof of Case 1 in Claim 3.1. In Case 1 , we do not have $A \cdot v=\mathrm{k} \cdot v$, in general. We have to change Case 1 and Case 2 into the following:

Case 1. Assume that $\operatorname{dim}_{\mathfrak{k}}\left(\operatorname{Soc}\left(A_{i}\right)\right) \geq 2$ for some $i$. Let $\mathfrak{n}_{1}, \mathfrak{n}_{2}$ be any two distinct minimal ideals in $\operatorname{Soc}\left(A_{i}\right)$. Since $\ell\left(A / \mathfrak{n}_{2}\right)<a+b+1$, it follows from assumption (A2) that $V_{1} V_{2}+\mathfrak{n}_{2}=A$. For any nonzero element $x$ of $\mathfrak{n}_{1}$, there exist elements $v \in V_{1} V_{2}$ and $y \in \mathfrak{n}_{2}$ such that $v+y=x$. Here note that $v \neq 0$, since $\mathfrak{n}_{1} \cap \mathfrak{n}_{2}=0$. Let $\mathfrak{n}^{\prime}$ be the ideal generated by $v$. Then $\ell\left(A / \mathfrak{n}^{\prime}\right)<a+b+1$ and $\mathfrak{n}^{\prime}=A \cdot v=\mathrm{k} \cdot v \subset V_{1} V_{2}$ since $v=x-y \in \operatorname{Soc}\left(A_{i}\right)$. Therefore by assumption (A2), we obtain

$$
A=V_{1} V_{2}+\mathfrak{n}^{\prime}=V_{1} V_{2} .
$$

Case 2. Assume that $\operatorname{dim}_{\mathrm{k}}\left(\operatorname{Soc}\left(A_{i}\right)\right)=1$ for all $i$. Then $A_{i}$ itself is a local ring, and $A_{i}$ has a unique minimal ideal $\mathfrak{n}_{i}$. It follows from [8, Theorem 221] that $A_{i}$ is a 0 -dimensional local Gorenstein ring. For any ideal $\mathfrak{a}$ of the direct product $A$ of 0 -dimensional local Gorenstein rings $A_{i}$, we obtain $(0:(0: \mathfrak{a}))=\mathfrak{a}$ by 7 , Exercise $3.2 .15]$, and

$$
\ell((0: \mathfrak{a}))=\ell(A / \mathfrak{a}) \quad \text { and } \quad \ell(\mathfrak{a})=\ell(A /(0: \mathfrak{a}))
$$

by considering the length of the composition series. Let $\mathfrak{b}, \mathfrak{c}$ be any ideals of $A$ such that $\mathfrak{b} \supset \mathfrak{c}, \ell(\mathfrak{b})=b+1$, and $\ell(\mathfrak{c})=b$. From $(3.2)$, we obtain $\ell(A /(0: \mathfrak{b}))=b+1$ and $\ell(A / \mathfrak{c})=a+1$. By the assumption of the claim, $V_{2}+(0: \mathfrak{b})=A$, and for any element $x \in(0: \mathfrak{c})$, there exist $v_{2} \in V_{2}$ and $z \in(0: \mathfrak{b})$ such that $v_{2}+z=x$. 
Similarly, $V_{1}+\mathfrak{c}=A$, and for any element $y \in \mathfrak{b}$, there exist $v_{1} \in V_{1}$ and $w \in \mathfrak{c}$ such that $v_{1}+w=y$. Then

$$
v_{1} v_{2}=(y-w)(x-z)=x y-y z-x w+z w=x y .
$$

Indeed, $y z \in \mathfrak{b} \cdot(0: \mathfrak{b})=0, w x \in \mathfrak{c} \cdot(0, \mathfrak{c})=0$, and $w z \in \mathfrak{c} \cdot(0: \mathfrak{b}) \subset \mathfrak{b} \cdot(0: \mathfrak{b})=0$. Hence we obtain

$$
(0: \mathfrak{c}) \cdot \mathfrak{b} \subset V_{1} V_{2} .
$$

Moreover $(0: \mathfrak{c}) \supsetneq(0: \mathfrak{b})$, and then $(0: \mathfrak{c}) \cdot \mathfrak{b} \neq 0$. Therefore by assumption (A2), we have

$$
A=V_{1} V_{2}+(0: \mathfrak{c}) \cdot \mathfrak{b}=V_{1} V_{2} .
$$

This completes the proof of the claim, hence Theorem 1.1.

\section{REFERENCES}

[1] E. Ballico and M. C. Beltrametti, On 2-spannedness for the adjunction mapping, Manuscripta Math. 61 (1988), 447-458. MR0952089 (89i:14003)

[2] M. C. Beltrametti, P. Francia and A. J. Sommese, On Reider's method and higher order embeddings, Duke Math. J. 58 (1989), 425-439. MR.1016428 (90h:14021)

[3] M. C. Beltrametti and A. J. Sommese, On $k$-spannedness for projective surfaces, Algebraic Geometry, Proc. L'Aquila 1988, Lecture Notes in Math. 1417, Springer-Verlag, 1990, pp.2451. MR1040549 (91g:14029)

[4] M. C. Beltrametti and A. J. Sommese, (with an appendix by L. Göttsche), Zero cycles and $k$-th order embeddings of smooth projective surfaces, Proc. Projective Surfaces and their Classification, Cortona 1988, Sympos. Math. 32, INDAM, Academic Press, 1992, pp.33-48. MR:1273371 (95d:14005)

[5] M. C. Beltrametti and A. J. Sommese, On k-jet ampleness, Complex Analysis and Geometry, Plenum Press, 1993, pp.355-376. MR.1211891 (94g:14006)

[6] M. C. Beltrametti and A. J. Sommese, On the preservation of $k$-very ampleness under adjunction, Math. Z. 212 (1993), 257-283. MR.1202811 (94a:14002)

[7] W. Bruns and J. Herzog, Cohen-Macaulay Rings, Cambridge Stud. Adv. Math. 39, Cambridge Univ. Press, 1993. MR1251956 (95h:13020)

[8] I. Kaplansky, Commutative Rings, Revised Edition, The Univ. of Chicago Press, 1974. MR0345945 (49:10674)

Department of Mathematics, School of Education, Waseda University, 1-6-1 NishiWASEda Shinjuku-Ku, TOKYO 169-8050, JAPAN

Current address: 389-3, Higashi-Koiso, Oiso-Mati, Naka-Gun Kanagawa, 255-0004, Japan

E-mail address: hinohara@waseda.jp

Department of Mathematics, School of Education, Waseda University, 1-6-1 NishiWaseda Shinjuku-Ku, Tokyo 169-8050, Japan

Current address: School of Social Sciences, Waseda University, 1-6-1 Nishi-Waseda Shinjukuku, Tokyo 169-8050, Japan

E-mail address: ktaka@asagi.waseda.jp

Tsuru University, 3-8-1 TAhara Tsuru-Shi, Yamanashi 402-8555, JAPAN

E-mail address: terakawa@tsuru.ac.jp 